\title{
Intracellular motor-driven transport of rodlike smooth organelles along microtubules
}

\author{
A. B. Fernández Casafuz $\odot,{ }^{1}$ M. C. De Rossi, ${ }^{2}$ and L. Bruno $\circledast^{1,3, *}$ \\ ${ }^{1}$ Instituto de Cálculo, Facultad de Ciencias Exactas y Naturales, Universidad de Buenos Aires, Argentina \\ ${ }^{2}$ Departamento de Química Biológica, Facultad de Ciencias Exactas y Naturales, Universidad de Buenos Aires, Argentina \\ ${ }^{3}$ Consejo Nacional de Investigaciones Científicas y Técnicas, Argentina
}

(Received 23 December 2019; revised manuscript received 19 February 2020; accepted 1 June 2020; published 19 June 2020)

\begin{abstract}
Molecular motors are fascinating proteins that use the energy of ATP hydrolysis to drive vesicles and organelles along cytoskeleton filaments toward their final destination within the cell. Several copies of these proteins bind to the cargo and take turns transporting the cargo attaching to and detaching from the track stochastically. Despite the relevance of molecular motors to cell physiology, key aspects of their collective functioning are still unknown. In this work we propose a one-dimensional model for the transport of extensive and smooth organelles driven by molecular motors. We ran numerical simulations to study the behavior of the cargo for different motor configurations, focusing on the transport properties observable in the experiments, e.g., average speed of the organelle and variations in length. We found that active motors drive the cargo using two different mechanisms: Either they locate in front of the cargo and pull the organelle or they situate at the cargo lagging edge and push. Variations in the organelle length is in close relation with the fraction of motors in each configuration, which depends on the resisting load. The results of this model were contrasted with experimental data obtained from the tracking of rodlike mitochondria during active transport in Xenopus laevis melanophores.
\end{abstract}

DOI: 10.1103/PhysRevE.101.062416

\section{INTRODUCTION}

The delivery of organelles, vesicles, and other cargoes toward their correct locations within cells requires the joint action of molecular motors [1]. These proteins use the energy provided by ATP hydrolysis to perform discrete nanometric steps along cytoskeleton filaments, i.e., microtubules and actin filaments, to displace cargoes processively. It has been suggested that the organization of motors on the surface of the cargo would be essential for the success of transport [2-6]. Also, recent works propose that certain properties of the organelle membrane, for example fluidity or smoothness, would modulate the mechanical interaction between motors and, therefore, the properties of transport [7-9]. However, very important aspects of the dynamic organization of motors on the organelle surface are still unknown.

Among intracellular cargoes are mitochondria; these organelles are present in most eukaryotic organisms and are responsible for the generation of the ATP required in numerous cellular processes (reviewed in Refs. [10,11]). Microtubule plus-end-directed motors kinesin- 1 and kinesin- 3 and the minus-end cytoplasmic dynein [12-14] attach to mitochondria through adaptor proteins such as the Miro-Milton complex and kinesin binding protein (KBP) (reviewed in Ref. [15]) to drive mitochondria along microtubules [16,17]. Molecular motors also play a relevant role in the regulation of mitochondrion shape and size $[13,14]$. It has been suggested that mitochondria change their morphologies to rodlike shape to facilitate their transportation [18], highlighting their flexibility.

\footnotetext{
*1bruno@df.uba.ar
}

Recently, we have studied the transport of rodlike mitochondria along microtubules and found a strong correlation between retrograde transport, i.e., toward the perinuclear region, and mitochondria retraction. We also found that most anterograde transported mitochondria, i.e., toward the cell cortex, preserved their size during transport suggesting that the mechanical communication among motors in minus-end and plus-end teams is different [19]. In a new paper, we combined fluorescence fluctuations and single-particle tracking techniques to explore the dynamics of EGFP-tagged kinesin-1 motors during active transport of mitochondria along microtubules in Drosophila melanogaster $\mathrm{S} 2$ cells [20]. We showed that kinesin exhibits different dynamics at the leading and at the rear regions of mitochondria, which in turn depends on the organelle direction of motion, highlighting the complex organization of motors during collective transport. Unfortunately, methodologies capable of addressing the behavior of individual motors within living cells are still lacking.

In this context, numerical simulations constitute useful tools that allow approaching the problem from a complementary perspective and exploring the organization of motors and their force production while transporting organelles. Several models of the motor-driven cargo transport have been proposed in the literature [21-26]. However, it should be noted that those models have limited their results to rigid and spherical organelles, which do not allow exploring the distribution of the motors on the organelle membrane or assay the effects of the localized forces exerted by the motors on the organelle surface that could lead to variations in the shape of the cargo.

Mitochondria and other intracellular organelles are surrounded by lipid membranes. These membranes can deform 
in different ways; for instance, they stretch due to tangential forces or they can change their curvature due to bending exerted by torques. In particular, the mechanical resistance to deformation by stretching has been extensively modeled as an elastic element (i.e., spring) with an associated stiffness [27-29]. On the other hand, more recently it has been determined that the viscosity of mitochondria would play an important role in its physiology [30-32]. These papers have highlighted the relevance of incorporating the rheological aspects in modeling membrane mechanics.

In this work, we propose a one-dimensional (1D) model of the transport of a long and smooth organelle driven by molecular motors along a microtubule. We explore in detail the distribution of motors on the organelle and the forces produced during the transport. We found that motors use two different configurations to transport extensive cargoes depending on the opposite load. While for large resisting loads they locate in front of the cargo and pull the organelle, for low values of the load they situate at the cargo lagging edge and push. Our model gives an interpretation of the deformations of transported mitochondria observed in living cells [19].

The paper is organized as follows: In Secs. II and III we present the model and details of the numerical simulations, respectively. Section IV describes the tracking experiments of mitochondria in living cells. The results are discussed in Sec. V. Finally, we present the conclusions in Sec. VI.

\section{THE MODEL}

We based our model on previous Langevin stochastic models $[23,25]$ where the cargo is driven by motors acting as parallel springs within a very viscous cytoplasm. The motors move stochastically in discrete nanometer steps along an unidimensional track, with load-dependent probabilities of stepping and detachment. Detached motors can rebind to the track with a constant probability or diffuse along the cargo. For typical values of cytosol viscosity and organelle sizes, inertial effects can be neglected, as discussed in previous works [33,34].

The novelty of the model proposed here is to consider an extensive and flexible cargo accounting for nonspherical organelles, such as mitochondria. It has been shown that the elastic behavior of isolated mitochondria is related to its membrane deformability and membrane tension [35]. Particularly, for the rod-shaped organelles driven by microtubule molecular motors along a 1D track considered in this paper, we proposed that stretching would be the main deformation mechanism that would lead to variations in the length of the organelle. Since it has been shown that the viscosity of mitochondria is very relevant for its physiology [30-32], we included it in the model. To this end, we assumed that the cargo has an internal structure composed of two masses, i.e., nodes, connected by an overdamped spring. Motors can attach to the microtubule and to either of these nodes and perform discrete steps along the track, hence exerting local forces on the cargo when their stretching exceeds their natural length. A scheme of the model is shown in Fig. 1.

We introduced two variables to describe the motion of the system: the position of the cargo center of mass $\left(X_{\text {c.m. }}\right)$ and its

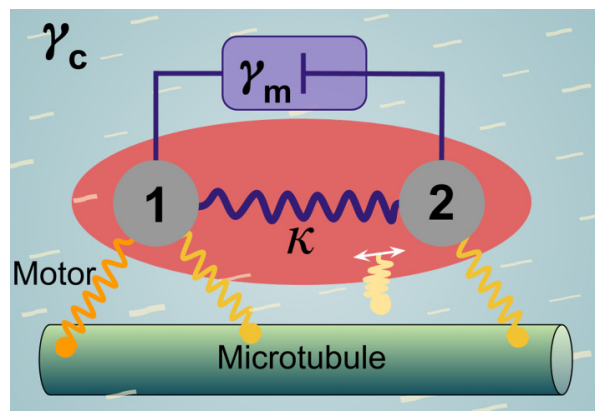

FIG. 1. The model: The cargo consists of two nodes, elastically coupled. Motors can attach to these nodes and perform discrete steps along the track. When unbound from the track, motors diffuse along the cargo.

length variation, or deformation $\left(X_{\text {def }}\right)$, which are defined as:

$$
\begin{gathered}
X_{\text {c.m. }}(t)=\frac{1}{2}\left[X_{1}(t)+X_{2}(t)\right] \\
X_{\operatorname{def}}(t)=X_{2}(t)-X_{1}(t)-L_{x},
\end{gathered}
$$

where $X_{1}(t)$ and $X_{2}(t)$ are the coordinates of the nodes at time $t$ and $L_{x}$ is the natural length of the spring that couples the nodes, which was set equal to $3 \mu \mathrm{m}$. Then the cargo transport and its dynamical deformation are described by the following coupled Langevin equations:

$$
\begin{gathered}
\gamma_{c} \frac{d X_{\text {c.m. }}}{d t}=\sum_{i=1}^{N} f_{i}^{1}(t)+\sum_{i=1}^{N} f_{i}^{2}(t)+\zeta_{c}(t), \\
\gamma_{m} \frac{d X_{\text {def }}}{d t}=-\kappa X_{\text {def }}+\sum_{i=1}^{N} f_{i}^{2}(t)-\sum_{i=1}^{N} f_{i}^{1}(t)+\zeta_{m}(t),
\end{gathered}
$$

where $N$ is the number of motors and $\sum f_{i}^{1}$ and $\sum f_{i}^{2}$ represent the total force exerted by the motors on nodes 1 and 2 , respectively. Individual motors are independent, but they can interact mechanically with other motors through the tension induced on the nodes, from here on called load. Although not considered here, the addition of an external force to the equations is straightforward.

Thermal activity is considered by the thermal noise $\zeta(t)$, according to the fluctuation-dissipation theorem [36,37]. Briefly, the thermal noise $\zeta(t)$ is a zero-centered random force with correlation function:

$$
\left\langle\zeta(t) \zeta\left(t^{\prime}\right)\right\rangle=2 \gamma k_{B} T \delta\left(t-t^{\prime}\right),
$$

where $k_{B}$ is the Boltzmann constant, $T$ is the absolute temperature, and $\delta(t)$ is the Dirac $\delta$ function. Finally, $\gamma$ is a drag coefficient related to the Stokes viscous force, i.e., left-hand sides in Eqs. (1) and (2). We called $\zeta_{c}$ and $\zeta_{m}$ the thermal noise in Eq. (1) and Eq. (2) to explicitly account for the differences between the drag coefficient in the cytosol and in the membrane, respectively.

These coupled equations can be interpreted as follows: On the one hand, Eq. (1) considers the motion of an equivalent rigid organelle, described by the cargo center of mass, in a viscous fluid, i.e., the cytosol, with a drag coefficient $\gamma_{c}$, and driven by the net force exerted by the motors. On the other 


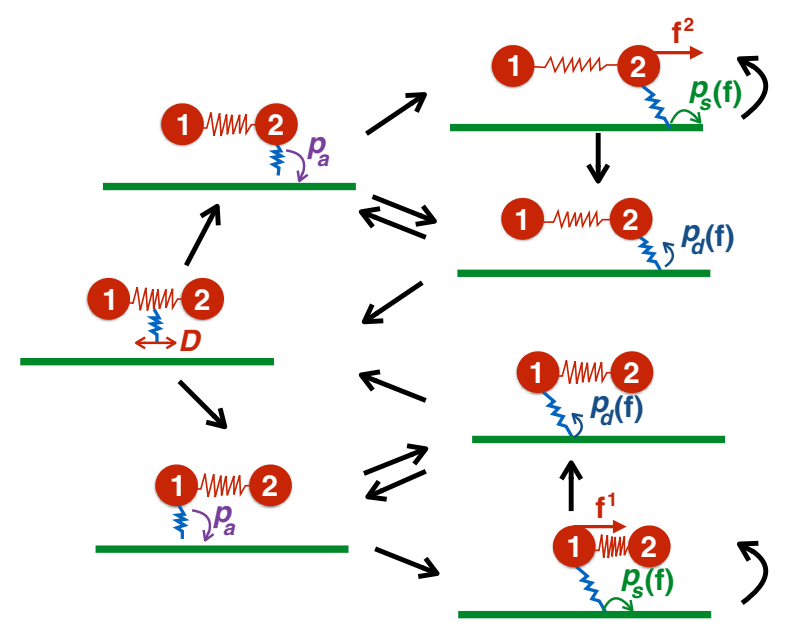

FIG. 2. Schematic of the single motor states and transitions. Black arrows represent the possible transitions between states. Motors diffuse while detached from the track. They can bind to node 1 or node 2. Attached motors can either detach from the track (and node) or perform discrete steps along the MT. Motors can exert forces on the node they are attached to only when they are stretched above their natural length.

hand, Eq. (2) describes the cargo extension and retraction dynamics caused by the opposing motor forces; in this equation, $\gamma_{m}$ represents the drag coefficient due to the viscous vesicle membrane and $\kappa$ is related to the membrane rigidity. In other words, the imbalance of the forces applied to nodes 1 and 2 is responsible both for the cargo motion along the track through Eq. (1) and for the cargo length variation through Eq. (2).

\section{NUMERICAL SIMULATIONS}

We used Euler's finite difference method in a Matlab environment to integrate Eqs. (1) and (2). We considered teams of $N$ motors acting on the cargo alone or combined in a tugof-war (TOW) scenario, i.e., competing against an opposite polarity team. Each motor follows a Monte Carlo procedure in an independent simulation routine for determining its coordinate at each time step. In Fig. 2 we represent a scheme of the motor states and the possible transitions between them.

Briefly, each unbound motor diffuses along the cargo with a diffusion coefficient $D$. We consider reflective boundary conditions for the diffusing motors, i.e., motors reaching the tip of the cargo are reflected. Diffusing motors redistribute along the cargo but cannot exert forces on the latter. These motors can attach to a node (1 or 2 ) and to the microtubule with a constant rate $p_{a}$. When a motor anchors to the node it is not allowed to diffuse from this site in the membrane. An attached motor detaches from the microtubule with a probability per time given by $[23,38]$ :

$$
p_{d}(f)=\epsilon_{0} e^{\frac{f}{F_{d}}}
$$

where $\epsilon_{0}$ is the detachment rate, $F_{d}$ is the detachment force of the motor, and $f$ is the absolute value of the load. Other mechanisms for the detachment kinetics other than Eq. (4) have been suggested for dynein [38]. However, there is not a general consensus and further experiments are needed to elucidate the behavior of dyneins under load. Also, a bound motor performs discrete 8-nm steps toward the plus-end (kinesins) or the minus-end (dyneins) of the microtubule with a probability per unit time equal to $[21,22,24]$ :

$$
p_{s}(f)=\left\{\begin{array}{ll}
v_{0}\left[1-\left(\frac{f}{F_{s}}\right)^{w}\right] & f<F_{s} \\
0 & f \geqslant F_{s}
\end{array},\right.
$$

where $F_{s}$ is the stall force and $v_{0}$ is the zero-load velocity. When an attached motor stretches, either because it performs a step or it is pulled by the moving cargo, it exerts a force $f_{i}^{j}$ on the corresponding node $j(j=1,2)$ given by [25]:

$$
f_{i}^{j}= \begin{cases}k\left(d_{i}^{j}-l_{0}\right) & d_{i}^{j}>l_{0} \\ 0 & -l_{0} \leqslant d_{i}^{j} \leqslant l_{0}, \\ k\left(d_{i}^{j}+l_{0}\right) & d_{i}^{j}<-l_{0}\end{cases}
$$

where $k$ represents the motor resistance to stretch beyond its natural length $l_{0}$ and $d_{i}^{j}$ is the distance between the motor position $a_{i}$ and the corresponding node position $X_{j}: d_{i}^{j}=a_{i}-$ $X_{j}$. When the force exerted by a motor is greater than $10^{-5} \mathrm{pN}$, it is considered an active motor. It is worth mentioning that not all motors are active simultaneously; the fraction of active motors is around $30 \%$ for single kinesins teams and $50 \%$ for dyneins.

We performed simulations of $200 \mathrm{~s}$ duration with a time step of $1 \mathrm{~ms}$. The numerical time step of $1 \mathrm{~ms}$ was determined as the most appropriate taking into account the optimization between the experiments duration and the computational cost; this was assessed in preliminary simulations using time steps in the range $1 \mu \mathrm{s}-10 \mathrm{~ms}$. Initially, all the motors were detached from the track and located at $X_{\text {c.m. }}$. After each simulation step, we computed the motors positions, the total force acting on each node and the number of active motors and obtained the cargo center of mass position $X_{\mathrm{c} . \mathrm{m} \text {. }}$ and length variation $X_{\text {def }}$ solving Eqs. (1) and (2). These magnitudes were resampled to $1 \mathrm{~s}$ (i.e., the experimental sampling time) and analyzed following the procedures used for those obtained experimentally (see below). The parameters used in the simulations are displayed in Table I.

Since we did not find experimental studies or models accounting for values of mitochondria internal stiffness, we decided to explore the behavior of the system for $\kappa$ between $3 \times 10^{-4}$ and $3 \times 10^{-2} \mathrm{pN} / \mathrm{nm}$. The largest value in the range corresponds to the stiffness of a motor-linker complex reported in Refs. [25,39] and considers the elastic behavior of adaptor proteins that link motors to the cargo membrane. On the other hand, the lower limit is in agreement with Jin et al. [40]; these authors studied the confined diffusion of membrane proteins and determined membrane rigidity values in the range $10^{-4}-10^{-3} \mathrm{pN} / \mathrm{nm}$.

\section{EXPERIMENTS}

The dynamics of mitochondria was studied in Xenopus laevis melanophores expressing the Xenopus homolog of $\tau$ protein XTP fused to Enhanced Green Flourescent Protein (EGFP), which associates with microtubules. These cells are relatively big $(\sim 50 \mu \mathrm{m})$ and thin $(\sim 3 \mu \mathrm{m}$, Ref. [6]); therefore, mitochondria displacement in the cell periphery is essentially bidimensional. We recovered confocal images 
TABLE I. Parameter values used in the simulations.

\begin{tabular}{lcc}
\hline \hline Magnitude & Symbol & Kinesin/dynein \\
\hline Cytosol viscosity $\left(\mathrm{pN} \mathrm{s} / \mathrm{nm}^{2}\right)$ & $\eta_{c}$ & $500 \eta_{\text {water }}$ \\
Thermal energy $(\mathrm{pN} \mathrm{nm})$ & $k_{B} T$ & 4 \\
Organelle width $(\mathrm{nm})$ & $\mathrm{W}$ & 600 \\
Organelle length $(\mathrm{nm})$ & $L_{o}$ & 3600 \\
Cytosolic drag coeff. $(\mathrm{pN} \mathrm{s} / \mathrm{nm})$ & $\gamma_{c}$ & 0.0071 \\
Membrane drag coeff. $(\mathrm{pN} \mathrm{s} / \mathrm{nm})$ & $\gamma_{m}$ & $2 \gamma_{c}$ \\
Membrane stiffness $(\mathrm{pN} / \mathrm{nm})$ & $\kappa$ & $3\left(10^{-4}-10^{-2}\right)[39,40]$ \\
Diffusion coefficient $(\mathrm{nm} / \mathrm{s})$ & $D$ & $0.5 \times 10^{6}[8,41]$ \\
Motor natural length $(\mathrm{nm})$ & $l_{0}$ & $100[20,22]$ \\
Motor stiffness $(\mathrm{pN} / \mathrm{nm})$ & $k$ & $0.03[39]$ \\
Step length $(\mathrm{nm})$ & $\Delta x$ & $8[42]$ \\
Zero-load velocity $(\mathrm{nm} / \mathrm{s})$ & $v_{0}$ & $500[43-45]$ \\
Exponent of F-V relation & $w$ & $2 / 0.5[46]$ \\
Stall force $(\mathrm{pN})$ & $F_{s}$ & $5.8 / 1.5[47-49]$ \\
Detachment rate $(1 / \mathrm{s})$ & $\epsilon_{0}$ & $1.1 / 0.2[50,51]$ \\
Detachment force $(\mathrm{pN})$ & $F_{d}$ & $3 / 0.8[22]$ \\
Binding rate $(1 / \mathrm{s})$ & $p_{a}$ & $2.6 / 5[50,52]$ \\
\hline \hline
\end{tabular}

of mitochondria labeled with MitoTracker Deep Red FM in actin-depolimerized melanocytes to ensure that transport only occurs along the microtubules. In those conditions, we have demonstrated that these organelles are in close proximity with these filaments [19]. Although these organelles exhibit a varied distribution of sizes and shapes (see Fig. 1 in Ref. [19]), in this work we focus on the mitochondria that have the following geometric restrictions: They are rod shaped and their trajectories are limited to 2D. We performed time-lapse experiments and applied a custom-made tracking routine to recover the trajectories of the mitochondria moving along microtubules with high spatial precision. Briefly, the centroid of rodlike mitochondria and its main axis length were determined in each frame of the movies to obtain the organelles trajectories and sizes, respectively (see Sec. VIB). The trajectories were fitted with a second-order polynomial function to define the mean transport direction and further decomposed to compute the distance traveled along the filament as a function of time. In order to assay changes in mitochondria size during transportation, we considered three cases, depending on the length variation $\Delta L$ during individual runs: extension for $\Delta L>100 \mathrm{~nm}$, retraction for $\Delta L<$ $-100 \mathrm{~nm}$, and no change in any other case. We analyzed 86 mitochondria from 35 cells.

\section{RESULTS AND DISCUSSION}

\section{A. Active transport of mitochondria in X. laevis melanocytes}

We tracked rodlike mitochondria in Xenopus laevis melanocytes [Figs. 3(a) and 3(b)] treated with Latrunculin, a drug that depolymerizes actin filaments. We recovered the organelles trajectories and their length simultaneously, following the same procedure as in [19]. Mitochondria moved along curvilinear microtubules following an almost onedimensional track. Individual trajectories displayed the typical bidirectional motion observed for mitochondria and other cargoes moving along microtubules [1] and involved periods of processive motion and frequent switches of direction
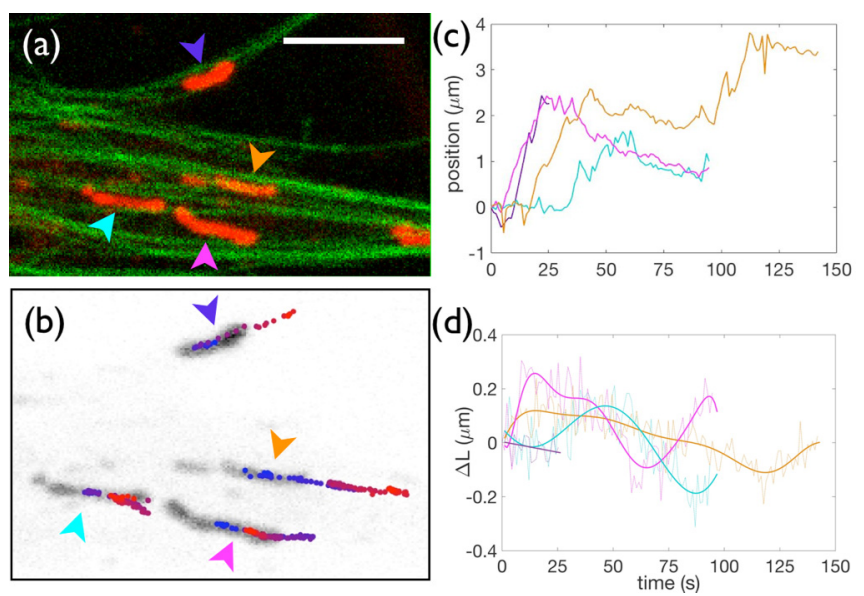

FIG. 3. (a) Two-channels confocal image showing microtubules (green) and mitochondria (red) in a $X$. laevis melanophore cell. Scale bar $=5 \mu \mathrm{m}$. (b) Grayscale image of the example shown in (a) overlapping the recovered trajectories of the mitochondria indicated with the colored arrow heads. The color scale from blue to red in the trajectories indicates time evolution. (c) Recovered position along the longitudinal direction and (d) length variation for the four mitochondria shown in (b). The filled lines in (d) correspond to a smoothing of the data (dashed lines) to facilitate visualization; $\Delta L=L-L_{o}$.

[Fig. 3(c)]. We found that mitochondria retracted, extended or preserved their size while being transported along microtubules [Fig. 3(d)].

To simplify the analysis of the data, we split trajectories into periods where the organelle moves processively, called runs. The length of the organelles at the beginning of each run, $L_{o}$, varied in the range $0.5-10 \mu \mathrm{m}$, with an average value equal to $3.6 \mu \mathrm{m}$. During runs mitochondria move with speeds less than a few hundreds of $\mathrm{nm} / \mathrm{s}$ (Fig. 4) in agreement with reported values in the literature $[53,54]$. We observed that mitochondria that lengthen were almost still or moved slower than mitochondria that preserved their size, according to a Kruskal-Wallis test [55] (asterisk in Fig. 4).

On the other hand, the mean relative extension or retraction of mitochondria, $\Delta L / L_{o}$ was around the $20-30 \%$ of the organelle length (Fig. 4).

\section{B. Model results: Identical motors teams}

Kinesin and dynein motor proteins transport the cargo toward the plus-end and minus-end of the microtubule, respectively. These two families of motors differ not only in their direction of motion but also in other important biophysical properties, such as the attachment and detachment rates and kinetics, as briefly discussed above. In this section, we consider identical motor teams composed of a different number of copies.

We first explored the effect of varying the organelle's stiffness $\kappa$ on the model behavior. To this end, we simulated trajectories of elongated organelles considering teams of equal polarity motors, i.e., kinesins or dyneins, using the parameters values displayed in Table I. Figure 5(a) shows representative model outputs for two kinesin teams consisting of 2 and 10 

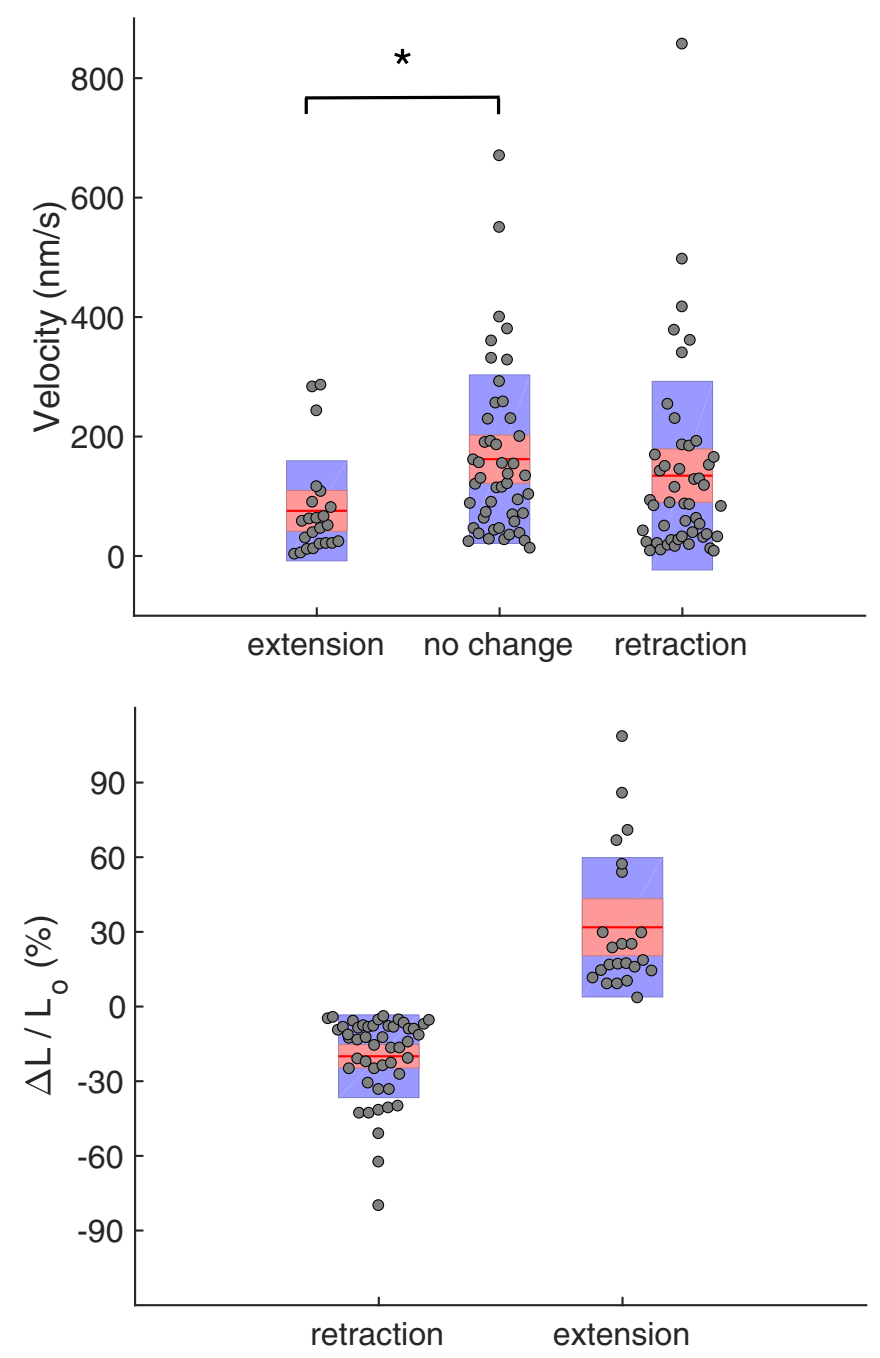

FIG. 4. Experimental results. Upper panel: Mitochondria velocities during runs where the organelle length increases $(N=23)$, does not change $(N=47)$, or decreases $(N=48)$. The mean velocities of the three sets are as follows: 75,160 , and $135 \mathrm{~nm} / \mathrm{s}$, respectively. The asterisk denotes significant differences between populations ( $p$ value $=0.004)$. Lower panel: Mitochondria relative length variation during runs where the organelle retracts $(N=48)$ or extends $(N=$ 23). Boxplots are presented as mean, SEM, and SD, according to Ref. [56].

motor copies, respectively, and $\kappa=0.003 \mathrm{pN} / \mathrm{nm}$. As it can be observed, in both cases the organelle moves processively with a mean speed around 100-350 nm/s, which is higher for the larger team. On the other hand, the cargo length fluctuates, displaying periods of stretching and retraction, with a predominance of contraction that is less pronounced when the number of motor copies in the team is reduced [inset in Fig. 5(a)]. This stems from the fact that, in the absence of opposite load, motors are located preferentially at the lagging part of the cargo and push the organelle, as will be further discussed below. Moreover, the organelle retraction fraction decreases for stiffer organelles [Figs. 5(b) and 5(d)]: As expected, less rigid organelles warp more than stiffer ones.

Following the procedures described above for the experimental data, we computed the average velocity of the cargo
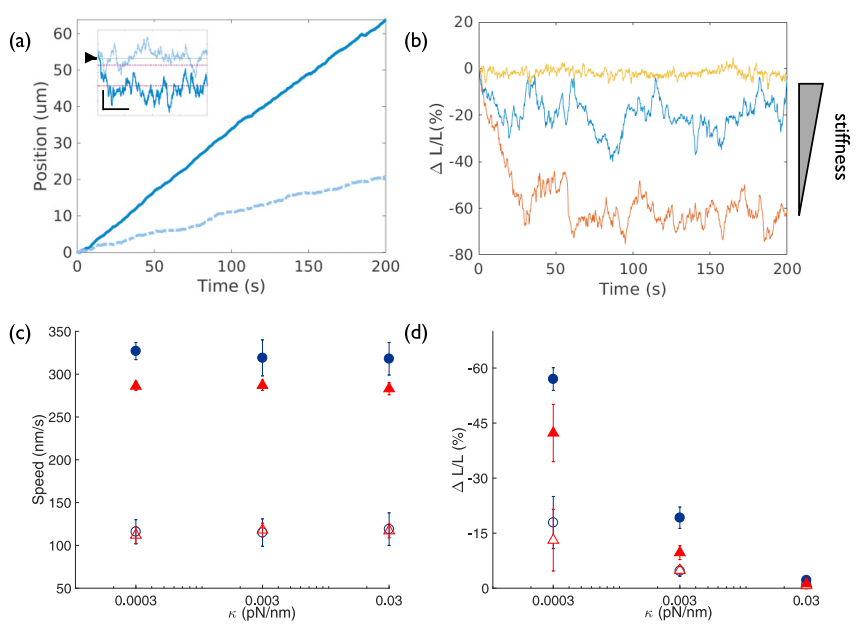

FIG. 5. Simulation results for identical motors teams. (a) Cargo trajectories and relative length variation (inset) for 2 (slashed line) and 10 (filled line) kinesin motor copies, $\kappa=0.003 \mathrm{pN} / \mathrm{nm}$. Scale bars in the inset: $500 \mathrm{~nm}$ and $50 \mathrm{~s}$. The arrowhead indicates $\Delta L=0$. (b) Organelle retraction fraction for 10 kinesin motor copies and decreasing stiffness values: $\kappa=0.03,0.003$, and $0.0003 \mathrm{pN} / \mathrm{nm}$. (c) Speed and (d) retraction fraction versus cargo stiffness for different motor teams: Kinesin (circles) and dynein (triangles). Open and filled symbols represent teams with 2 and 10 motor copies, respectively.

and its relative length variation for different number of motors and cargo stiffness. As expected, the cargo speed is insensitive to the value of $\kappa$ but depends on the number of motor copies and the biophysical properties of the motors driving the organelle (Fig. 5). A closer look to Figs. 5(c) and 5(d) shows that the model simulations with cargo stiffness in the range $0.0003-0.003 \mathrm{pN} / \mathrm{nm}$ and 2 to 10 motor copies mimic the experimental values obtained for mitochondria velocities and length variations in X. laevis melanocytes (Fig. 4). Furthermore, these $\kappa$ values are consistent with the stiffness reported for membrane receptors in mammalian cells [40]. Thus, we decided to further explore the model setting $\kappa=$ $0.003 \mathrm{pN} / \mathrm{nm}$.

To analyze how motors distribute, we first determined their relative localization along the organelle. Since the cargo changes its length in an stochastic manner while moving along the track, we defined the dimensionless relative position of each motor at time $t_{s}$ as:

$$
\tilde{x}_{i}\left(t_{s}\right)=\frac{x_{i}\left(t_{s}\right)-X_{1}\left(t_{s}\right)+r}{L\left(t_{s}\right)},
$$

where $x_{i}\left(t_{s}\right)$ and $X_{1}\left(t_{s}\right)$ are the coordinates of the $i$ th motor and node 1 at time $t_{s}$, respectively, $r$ is the node radius, and $L\left(t_{s}\right)$ is the cargo length, computed as $L\left(t_{s}\right)=L o+X_{\mathrm{def}}\left(t_{s}\right)$. These new coordinates take values between 0 and 1 , where 0 represents the lagging edge of the organelle and 1 stands for its leading part, for kinesins, while for dyneins 0 and 1 represent the leading and lagging edge, respectively.

Then, we computed the probability density estimate of the dimensionless position of the motors using the ksdensity function in Matlab. In Fig. 6(a) we show that the distribution of motors is not homogeneous but displays an increase in the 

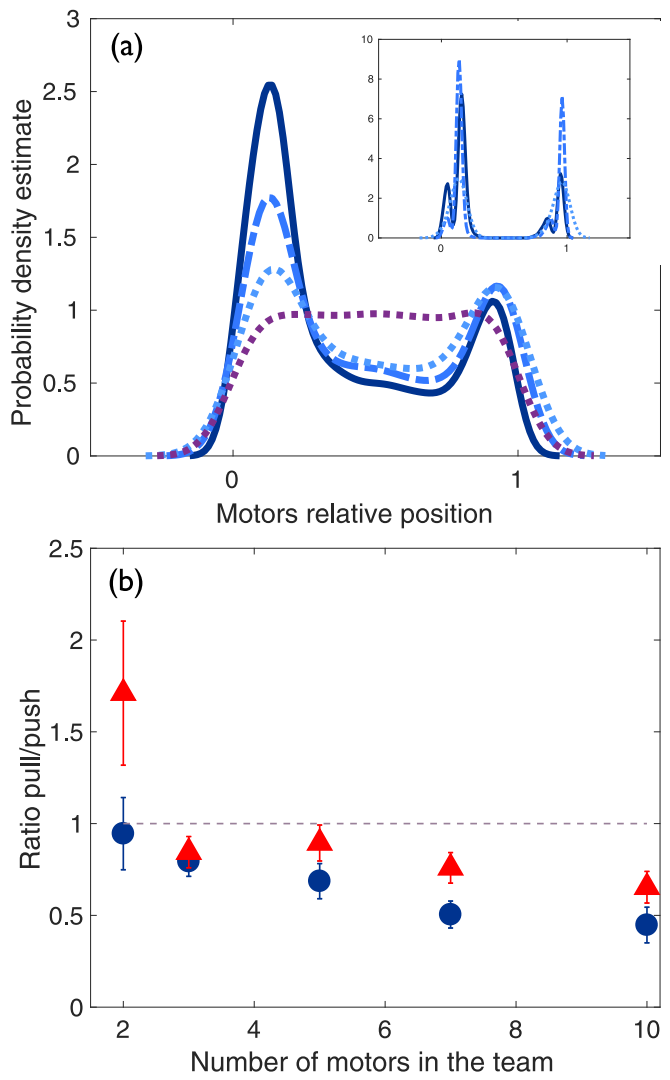

FIG. 6. Simulations: (a) Motors distribution along the organelle are represented by a probability density estimate for kinesin teams with 10 (filled line), 3 (dashed line), and 2 (dotted line) motor copies. Dark dotted line is the distribution of a 10 kinesins team where motors are unable to attach to the track, so they only diffuse along the cargo. Inset: Distribution of active motors in the corresponding cases. (b) Pulling motors vs. Pushing motors relative populations as a function of the number of motors in the team for kinesin (blue circles) and dynein (red triangles).

density at the organelle edges. To rule out that these peaks do not constitute an artifact of the cargo boundary condition, we considered a team of 10 motors that diffused along the cargo but were unable to bind to the track. This was done by setting the motors attachment probability equal to zero in the simulations. In this case, the peaks faded and we obtained a homogeneous distribution, supporting the conclusion that the enhanced motor concentration at the cargo edges is due to the motors attached to the track, as illustrated in the model scheme (Fig. 1). Furthermore, the active motors distribute within a narrow region around the position of the nodes [Fig. 6(a) (inset)]. This is not surprising since in our model motors can only attach to the track when they are close enough to a node; otherwise, they diffuse along the cargo. However, we found an asymmetry between the motors rear location and the forward one, which is caused by the prevalence of active motors in the trailing part of the organelle as shown in Fig. 6(a). This effect is more prominent for larger teams, where also the number of active motors involved in the force production increases, accounting for the increment in the contraction of the organelle for numerous teams [Fig. 5(d)]. (a)
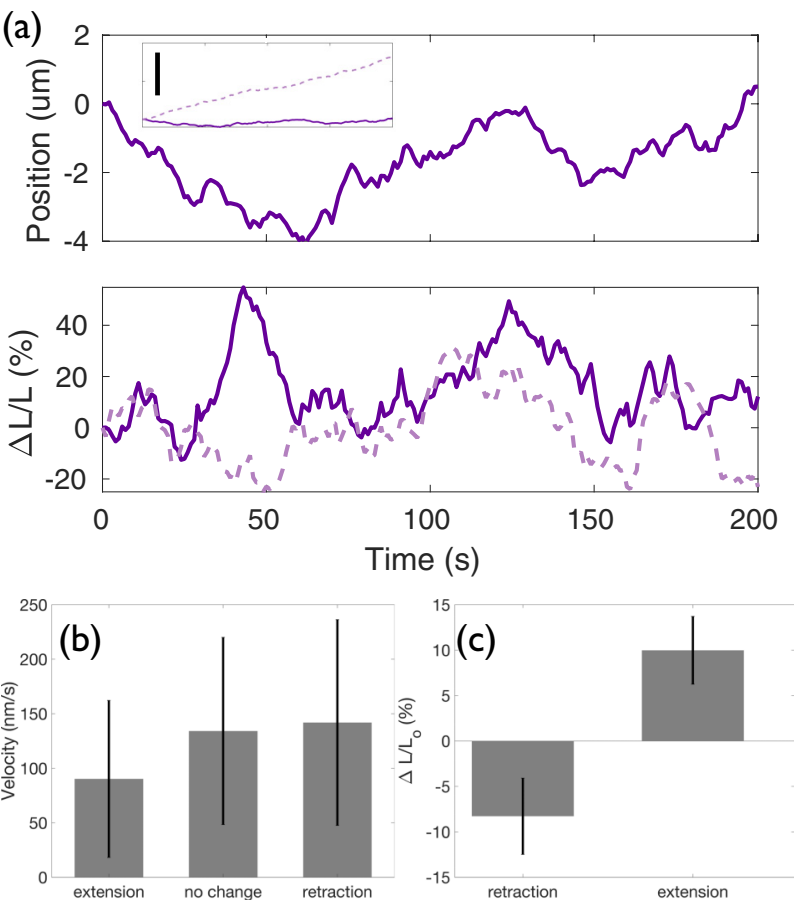

FIG. 7. Simulations: (a) Representative cargo trajectories (upper panel) and the corresponding length variation (lower panel) for two tug-of-war scenarios 10 kinesin vs. 9 (filled line) and vs. 5 (dashed line) dyneins. Scale bar in the upper panel: $20 \mu \mathrm{m}$. (b) Mean cargo velocity during extension $(90 \pm 72 \mathrm{~nm} / \mathrm{s})$, retraction $(142 \pm$ $94 \mathrm{~nm} / \mathrm{s})$, and no length variation $(134 \pm 86 \mathrm{~nm} / \mathrm{s})$ events in the $10 \mathrm{k}-$ 9d scenario. (c) Mean length variations for retracting and extending cargoes for the case shown in (b). Error bars computed as \pm SD.

To quantify this asymmetry in the motors distribution, we evaluated the ratio between the motors located at the leading edge, i.e., pulling motors, and the motors at the rear edge, i.e., pushing motors. This ratio was computed as the quotient between the two local maximums of the density at the leading and rear edges, respectively. In analogy with the vehicular transport, a ratio value less that 1 would describe a rearwheel drive (RWD) while the opposite case would represent a front-wheel drive. Our results show that for identical motor teams, both kinesins and dyneins assume a rear-wheel drive collective behavior for teams with more that two motors [Fig. 6(b)].

\section{Model results: Opposite polarity motor teams}

We next explored the behavior of the cargo in the presence of two opposite polarity motor teams: kinesins vs. dyneins. In order to explore scenarios with varying degrees of competition, i.e., tug-of-war, we ran simulations of 30 pairs of teams composed with different number of motor copies in the range $0-15$. Figure 7 (a) shows two trajectories representing high (10 kinesins and 9 dyneins) and low (10 kinesins and 5 dyneins) competitive cases, respectively. It can be seen that the cargo switches frequently its direction of motion as a consequence of the tug-of-war between the opposite polarity teams. Furthermore, large stretching of the organelle is more frequent than in the single team case, where the cargo tends 

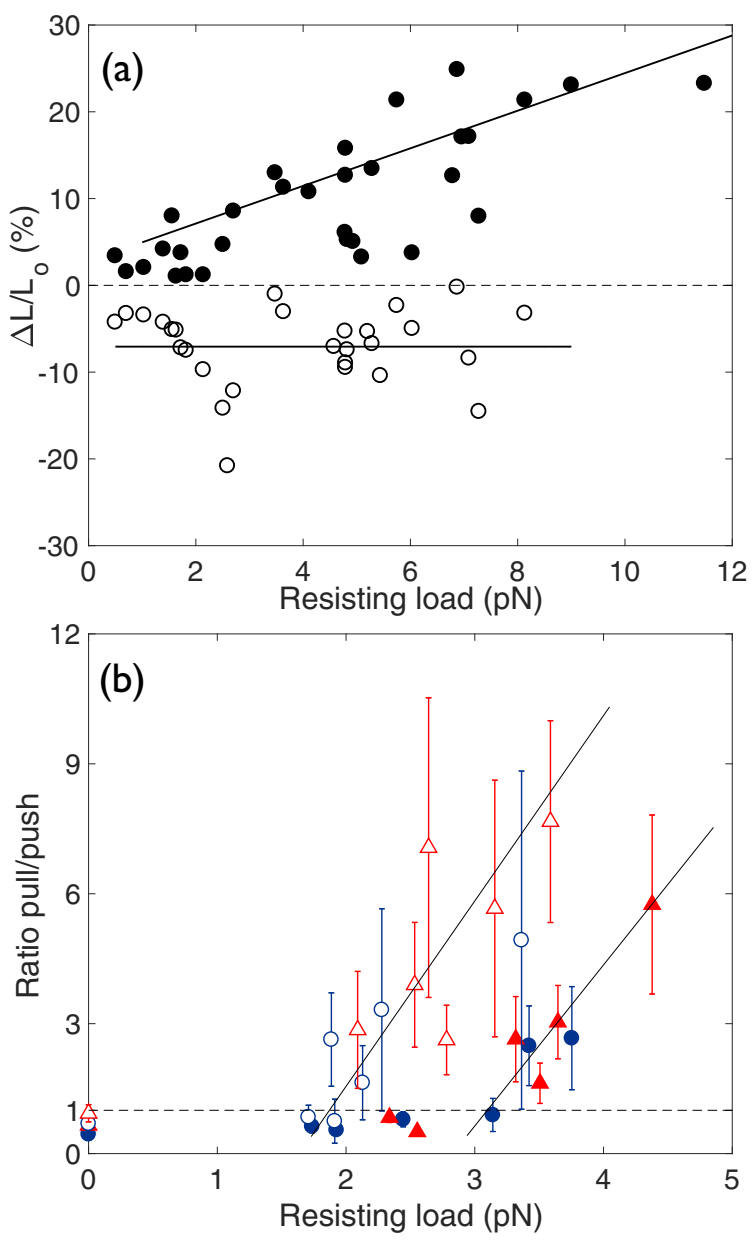

FIG. 8. Simulations. (a) Cargo relative length variation as a function of the resisting load for different tug-of-war scenarios. Data are split for extension (filled circles) and retraction (void circles) events. The filled lines are linear regressions of the data, with slopes $2.2 \pm 0.6$ and $0 \pm 0.03 \% / \mathrm{pN}$. (b) Ratio between pulling and pushing motors populations as a function of the resisting load assayed for 5 (void symbols) and 10 (filled symbols) motors teams. Circles and triangles stand for kinesin and dynein, respectively. Ratios are presented as means and SD of 10 estimations using samples of 100 data points each. The filled lines are guides to the eye.

to retract. The analysis of these data using the procedure described above for the experiments [Figs. 7(b) and 7(c)] showed that the velocities and deformations of the cargo are in agreement with the ones obtained for mitochondria in cells (Fig. 4). Even more, simulated organelles also tend to slow down when stretched as observed in the experiments.

We hypothesize that in a tug-of-war scenario each team confronts a resisting load exerted by the opposite polarity team, which increases with the number of active motors in the opposite team. To assess this hypothesis, we considered each of the teams (e.g., kinesins teams) and we computed the force produced by the opposite polarity team, i.e., dyneins; this was calculated averaging the net force produced by active dyneins on the cargo over the whole trajectory. In Fig. 8(a) we show that an increase in the resisting load is related with an increase in the cargo stretching. On the contrary, the retraction magnitude does not show an explicit dependence on the resisting load. Considering that extending organelles also move slower [Figs. 4 and 7(b)], we conclude that stretching configurations are associated with high competitive tug-ofwar.

Finally, we analyzed the distribution of motors along the cargo in the tug-of-war scenario. To this end, we computed the ratio between the pulling and pushing motors for kinesin and dynein in the different team configurations. We found that for low values of the resisting force, the motors distribute in a RWD fashion as in the case of single teams with 0 resisting load: i.e., they push the cargo predominantly. But as the resisting load increases, the motors invert their relative distribution and the pulling population surpasses the pushing one [Fig. 8(b)].

\section{CONCLUSIONS}

In this paper, we studied the collective dynamics of molecular motors while driving a rodlike and smooth cargo. Particularly, we explored how motors distribute along the cargo and exert forces able to extend or retract the cargo length. To this end, we developed a model including an internal degree of freedom for the cargo which accounts for its length variation; this was accomplished considering an overdamped spring connecting two nodes. Motors diffuse along the cargo length until they bind stochastically to the nearest node and the track, inducing tension on the cargo. We found that active motors drive the cargo using two different mechanisms: Either they locate in front of the cargo and pull the organelle or they situate at the cargo lagging edge and push. Numerical simulations of the model enabled studying the behavior of different motor teams in competitive and noncompetitive scenarios. Our results suggested that active motors adopt opposite configurations depending on the resisting load: For zero or small opposing load, they predominantly push the cargo; as a consequence the organelle contracts. On the other hand, when the resisting load is large (e.g., in very competitive tug-of-war with opposite polarity teams), motors pull the cargo and the organelle elongates.

The results of this model were contrasted with experimental data obtained from the tracking of rodlike mitochondria during active transport in X. laevis melanophores. In the experiments we observed a negative correlation between mitochondria speed and elongation, which is consistent with the differential motion of the organelle rear (fast) and front (slow) edges shown in our previous work [19]. In the mentioned work, we also found a strong correlation between retrograde transport and mitochondria retraction, whereas most anterograde mitochondria preserved their size during transport. These complex behavior can be interpreted in terms of our model: Retrograde transport would be mainly carried out by a team of several dyneins, in the absence of TOW, while anterograde motion would be subject to a greater degree of TOW or would consist of a smaller number of kinesin copies. This is consistent with the results showing that the cargo tend to contract for numerous teams of identical motors, while it elongates for TOW or less numerous teams.

Although here we explore the behavior of rod-shaped mitochondria during active transport, the model could also potentially be used to address the transport of tubular endo- 
somes [47] or the extraction of membrane tubes by kinesin engines [3]. Furthermore, the model might be extended to rigid and/or small cargoes by tuning some parameters values; for instance, increasing the membrane stiffness value would address the study of rigid organelles such us melanosomes [57], while shortening the natural length of the spring coupling the nodes could account for small particles, such as peroxisomes [58].

Finally, recent in vitro experiments using a novel forcegliding assay technique showed that kinesin motors dynamically interact inducing tension on the cargo [9]. The authors found that motors adopt two distinct states: resisting and pulling kinesins. In this context, our model also provides a theoretical interpretation to those results.

\section{ACKNOWLEDGMENTS}

This research was supported by Agencia Nacional de Promoción Científica y Tecnológica, Argentina (PICT 20150370). L.B. is member of CONICET.

\section{APPENDIX: MATERIALS AND METHODS}

\section{Cell culture and sample preparation for imaging}

Immortalized $X$. laevis melanophores stably expressing the Xenopus $\tau$-like protein XTP fused to EGFP $[59,60]$ were cultured as described in Ref. [61]. Cells were grown in $70 \%$ L-15 medium (Sigma-Aldrich) supplemented with phenylthiourea [62] to reduce the amount of melanosomes. For microscopy measurements, cells were grown for 2 days on $25-\mathrm{mm}$ round coverslips placed into $35-\mathrm{mm}$ plates in $2.0 \mathrm{ml}$ of complete medium. Before observation, the coverslips were washed in a serum-free $70 \%$ L-15 medium (SigmaAldrich) and mounted in a custom-made chamber specially designed for the microscope where the cells were incubated with $10 \mathrm{mM}$ of Latrunculin B (Sigma-Aldrich) for $30 \mathrm{~min}$ to depolymerize actin filaments. Mitochondria were labeled adding $100 \mathrm{nM}$ MitoTracker Deep Red FM (Invitrogen) to the incubation medium.

\section{Confocal imaging and tracking}

Confocal images were acquired in an FV1000 confocal microscope (Olympus Inc.). EGFP-XTP and MitoTracker Deep Red FM were observed using a multiline Ar laser tuned at 488-nm and a 635-nm diode laser as excitation sources (average power at the sample, 2 and $0.2 \mu \mathrm{W}$, respectively). The laser's light was reflected by a dichroic mirror (DM405/488/543/635) and focused through an Olympus UPlanSApo $60 \times$ oil immersion objective $(\mathrm{NA}=1.35)$ on to the sample. Fluorescence was collected by the same objective and split into two channels set to collect photons in the range 500-525 nm (EGFP) and 650-750 nm (MitoTracker Deep Red FM). Fluorescence was detected with photomultipliers set in the photon-counting detection mode. Time-lapse confocal movies $(100-150$ frames, pixel size $=63 \mathrm{~nm})$ of individual fluorescent mitochondria were collected at a speed of 0.6 frames/s. Custom made routines using MATLAB Image Processing Toolbox were used to recover the trajectories and lengths of the mitochondria from the fluorescence images. Each frame of the movie was processed individually, in a semiautomatic way. Briefly, the background was subtracted to each image and binarized. Then, for each region of interest in the image, i.e., mitochondria, the Centroid and the MajorAxisLength were computed using the MATLAB's regionprops function. The mitochondria positions and lengths were determined as the values obtained for the Centroid and "MajorAxisLength" parameters, respectively. According to the MATLAB Documentation page this last parameter returns the length (in pixels) of the major axis of the ellipse that has the same normalized second central moments as the region, returned as a scalar [63].
[1] W. O. Hancock, Nat. Rev. Mol. Cell Biol. 15, 615 (2014)

[2] S. Ally, A. G. Larson, K. Barlan, S. E. Rice, and V. I. Gelfand, J. Cell Biol. 187, 1071 (2009).

[3] O. Campàs, C. Leduc, P. Bassereau, J. Casademunt, J.-F. Joanny, and J. Prost, Biophys. J. 94, 5009 (2008).

[4] C. Leduc, O. Campàs, K. B. Zeldovich, A. Roux, P. Jolimaitre, L. Bourel-Bonnet, B. Goud, J.-F. Joanny, P. Bassereau, and J. Prost, Proc. Natl. Acad. Sci. USA 101, 17096 (2004).

[5] P. M. Shaklee, L. Bourel-Bonnet, M. Dogterom, and T. Schmidt, Biophys. J. 98, 93 (2010).

[6] M. C. De Rossi, L. Bruno, A. Wolosiuk, M. A. Despsito, and V. Levi, Biochim. Biophys. Acta 1830, 5095 (2013).

[7] A. Rai, D. Pathak, S. Thakur, S. Singh, A. K. Dubey, and R. Mallik, Roop, Cell 164, 722 (2016).

[8] S. R. Nelson, K. M. Trybus, and D. M. Warshaw, Proc. Natl. Acad. Sci. USA 111, E3986 (2014).

[9] M. Tjioe, S. Shukla, R. Vaidya, A. Troitskaia, C. S. Bookwalter, K. M. Trybus, Y. R. Chemla, and P. R. Selvin, eLife 8, e50974 (2019).

[10] D. C. Chan, Cell 125, 1241 (2006).

[11] A. J. Roger, S. A. Munoz-Gomez, and R. Kamikawa, Curr. Biol. 27, R1177 (2017).
[12] M. Nangaku, R. Sato-Yoshitake, Y. Okada, Y. Noda, R. Takemura, H. Yamazaki et al., Cell 79, 1209 (1994).

[13] D. G. Iworima, B. A. Pasqualotto, and G. L. Rintoul, Mol. Cell. Neurosci. 72, 22 (2016).

[14] A. Varadi, L. I. Johnson-Cadwell, V. Cirulli, Y. Yoon, V. J. Allan, and G. A. Rutter, J. Cell Sci. 117, 4389 (2004).

[15] R. L. Frederick and J. M. Shaw, Traffic 8, 1668 (2007).

[16] R. J. Youle and A. M. van der Bliek, Science 337, 1062 (2012).

[17] A. S. Moore and E. L. F. Holzbaur, Curr. Opin. Physiol. 3, 94 (2018).

[18] V. Anesti and L. Scorrano, Biochim. Biophys. Acta 1757, 692 (2006).

[19] M. C. de Rossi, V. Levi, and L. Bruno, Biosci. Rep. 38, BSR20180208 (2018).

[20] M. C. De Rossi, N. G. Bardeci, Y. Álvarez, E. Mocksos, J. J. Romero, L. Bruno, D. E. Wetzler, and V. Levi, Biochimica et Biophysica Acta (BBA) - Molecular Cell Research 1867, 118572 (2020).

[21] M. J. I. Müller, S. Klumpp, and R. Lipowsky, Proc. Natl. Acad. Sci. USA 105, 4609 (2008). 
[22] A. Kunwar, M. Vershinin, J. Xu, and S. P. Gross, Curr. Biol. 18, 1173 (2008).

[23] A. Kunwar and A. Mogilner, Phys. Biol. 7, 016012 (2010).

[24] A. Kunwar, S. K. Tripathy, J. Xu, M. K. Mattson, P. Anand, R. Sigua, M. Vershinin, R. J. McKenney, C. C. Yu, A. Mogilner, and S. P. Gross, Proc. Natl. Acad. Sci. USA 108, 18960 (2011).

[25] S. Bouzat, V. Levi, and L. Bruno, PLoS ONE 7, e43599 (2012).

[26] W. Nam and B. I. Epureanu, PLoS Comput. Biol. 11, e1003981 (2015).

[27] W. Helfrich, Z. Naturforsch C 28, 693 (1973).

[28] R. Hochmuth et al., Biophys. J. 13, 747 (1973).

[29] D. Boal, Mechanics of the Cell, 2nd ed. (Cambridge University Press, Cambridge, UK, 2012).

[30] Jiang et al., Sens. Actuat. B 190, 685 (2014).

[31] X. Song, N. Li, C. Wang, and Y. Xiao, J. Mater. Chem. B 5, 360 (2017).

[32] Zhang et al., J. Mater. Chem. B 7, 2749 (2019).

[33] S. Yamada, D. Wirtz, and S. C. Kuo, Biophys. J. 78, 1736 (2000).

[34] L. Bruno, V. Levi, M. Brunstein, and M. A. Desposito, Phys. Rev. E 80, 011912 (2009).

[35] Wang et al., Cell. Mol. Bioeng. 1, 67 (2008).

[36] R. Zwanzig, Nonequilibrium Statistical Mechanics (Oxford University Press, New York, 2001).

[37] R. K. Pathria, Statistical Mechanics (Pergamon Press, Oxford, 1972).

[38] Y. Ezber, V. Belyy, S. Can, and A. Yildiz, Nat. Phys. 16, 312 (2020).

[39] L. Bruno, M. Salierno, D. E. Wetzler, M. A. Despósito, and V. Levi, PLoS ONE 6, e18332 (2011).

[40] S. Jin, P. M. Haggie, and A. S. Verkman, Biophys. J. 93, 1079 (2007).

[41] R. Grover, J. Fischer, F. W. Schwarz, W. J. Walter, P. Schwille, and S. Diez, Proc. Natl. Acad. Sci. USA 113, E7185 (2016).

[42] A. G. Hendricks, E. L. Holzbaur, and Y. E. Goldman, Proc. Natl. Acad. Sci. USA 109, 18447 (2012).

[43] A. J. Hunt, F. Gittes, and J. Howard, Biophys. J. 67, 766 (1994).

[44] W. Nam and B. I. Epureanu, J. Phys.: Condens. Matter. 24, 375103 (2012).
[45] R. A. Longoria and G. T. Shubeita, PLoS ONE 8, e67710 (2013).

[46] A. K. Rai, A. Rai, A. J. Ramaiya, R. Jha, and R. Mallik, Cell 152, 172 (2013).

[47] V. Soppina, A. K. Rai, A. J. Ramaiya, P. Barak, and R. Mallik, Proc. Natl. Acad. Sci. USA 106, 19381 (2009).

[48] M. J. Schnitzer, K. Visscher, and S. M. Block, Nat. Cell Biol. 2, 718 (2000).

[49] V. Belyy, M. A. Schlager, H. Foster, A. E. Reimer, and A. Yildiz, Nat. Cell Biol. 18, 1018 (2016).

[50] R. Jiang, S. Vandal, S. Park, S. Majd, E. Tüzel, and W. O. Hancock, Proc. Natl. Acad. Sci. USA 116, 26564 (2019).

[51] C. M. Coppin, D. W. Pierce, L. Hsu, and R. D. Vale, Proc. Natl. Acad. Sci. USA 94, 8539 (1997).

[52] B. Gutiérrez-Medina, M. Buendía Padilla, A. J. GutiérrezEsparza, A. R. Oaxaca Camachoa, Biophys. Chem. J. 242, 28 (2018).

[53] J. G. Mc Carron, C. Wilson, M. E. Sandison, M. L. Olson, J. M. Girkin, C. Saunter, and S. Chalmers, J. Vasc. Res. 50, 357 (2013).

[54] L. C. Woods, G. W. Berbusse, and K. Naylor, Front. Cell Dev. Biol. 4, 19 (2016).

[55] We used the function kruskalwallis in MATLAB Statistics Toolbox.

[56] R. Campbell, GitHub: notBoxPlot (2019), https://www.github. com/raacampbell/notBoxPlot.

[57] S. Guo, L. Hong, B. B. Akhremitchev, B. B. and J. D. Simon, J. D, Photochem. Photobiol. 84, 671 (2008).

[58] A. Neuhaus, C. Eggeling, R. Erdmann, and W. Schliebs, Biochimica et Biophysica Acta (BBA) - Molecular Cell Research 1863, 1019 (2016).

[59] V. Levi, A. S. Serpinskaya, E. Gratton, and V. Gelfand, Biophys. J. 90, 318 (2006).

[60] O. F. Olesen, H. Kawabata-Fukui, K. Yoshizato, and N. Noro, Gene 283, 299 (2002).

[61] S. L. Rogers, I. S. Tint, P. C. Fanapour, and V. I. Gelfand, Proc. Natl. Acad. Sci. USA 94, 3720 (1997).

[62] S. P. Gross, M. C. Tuma, S. W. Deacon, A. S. Serpinskaya, A. R. Reilein, and V. I. Gelfand, J. Cell Biol. 156, 855 (2002).

[63] https://www.mathworks.com/help/images/ref/regionprops. html. 